\title{
Emulation Training System for Power Safety
}

\author{
Lanxiang Wang ${ }^{1, \text { a }}$, Jun Wang ${ }^{2}$, Hao Meng ${ }^{2}$, Ping $\mathrm{Li}^{2}$, Lei Zhou ${ }^{1}$, \\ Xuanhuai Yang ${ }^{1}$ and Guozhong Liu $^{2}{ }^{2 *}$ \\ ${ }^{1}$ Beijing Kedong Electric Power Control System Co., Ltd, Beijing 100192, China; \\ ${ }^{2}$ Beijing Information Science and Technology University, Beijing 100192, China.
}

alamne@163.com, ${ }^{\mathrm{b}}$ liuguozjong@bistu.edu.cn

*The corresponding author

\begin{abstract}
The traditional power safety training methods are difficult to achieve a good training effect. In this paper, an entity emulation system for power safety training based on the three-dimensional stereo vision and the wireless communication technology is presented. A real-time 3D motion capture unit based on stereo vision is used to acquire the space position of markers fixed on the power equipment emulators and on the clothes of training personnels to monitor the status of power equipment emulators and recognize the action behaviors of training personnels. Wireless network modules are used to transmit device status monitoring data from the power equipment emulators to the main computer, and to transmit control signals from the main computer to the power equipment emulators to simulate the device failure phenomenon in case of misoperation. The proposed entity emulation system can provide an effective, realistic and immersive training environment for power system personnel training.
\end{abstract}

Keywords: Power security training; Entity emulation; Motion capture; 3D stereo vision; Zigbee wireless network; Failure phenomenons emulation

\section{电力安全培训实体仿真系统}

\section{王兰香 ${ }^{1}$ ，王军 ${ }^{2}$ ，孟浩 ${ }^{2}$, 李萍 ${ }^{2}$, 周䂞 ${ }^{1}$, 杨选怀 ${ }^{1}$, 刘国忠 ${ }^{2, *}$}

（1. 北京科东电力控制系统有限责任公司，北京 100192；2．北京信息科技大学，北京 100192）

摘要: 传统的电力安全培训的方法很难达到良好的训练效果。文章提出了一种基于三维立体视觉、无线通信技术和电子 技术的电力安全培训实体仿真系统。基于立体视觉的三维运动捕获单元实时获取固定在仿真电力设备和操作人员工作服上的 标记点三维空间位置, 用于监测设备工作状态和识别操作人员动作行为。ZigBee 无线网络模块将设备状态监测数据从仿真 电力设备传送到主计算机, 并将主计算机发出的控制信号传送到仿真电力设备, 模拟工作人员误操作产生的各种故障现象。 所提出的实体仿真培训系统可实现有效的, 真实的和身临其境的电力设备操作安全培训。

关键词: 电力安全培训; 实体仿真; 动作捕捉; 三维立体视觉; ZigBee 无线传感网络; 故障现象模拟

中图分类号：TM 08 文献标志码：A

\section{引言}

电力安全生产是涉及职工生命安全的大事, 也是影响电力系统安全、稳定运行和电力企业生存发展的 关键 ${ }^{[1]}$ 。由于电力系统的特殊性, 对作业人员的操作技能要求越来越高, 为此电力企业投入了大量的人力、 物力、财力, 来提高电力职工队伍的素质和安全生产意识。传统的电力安全培训方式主要是举办集中培训 班、研讨会等, 采取理论讲解、多媒体课件等教学形式, 定期组织学员到作业现场进行观摩和实习, 提高 培训学员的感性认识和动手能力。但这些方式成本高、形象性差, 培训效果不明显。现场培训虽然效果良 好, 但由于很多设备是带电运行, 存在危险性和不确定性, 在实际运行的设备中进行人员培训具有很大的 
局限性。

利用计算机软件技术开发的仿真培训系统使学员有身临其境的感受, 对学员进行高效培训, 并进行操 作及理论考核, 提高培训效率。软件仿真主要以接线图、数字图表、设备照片和现场录像方式等常规多媒 体方式作为表现手段, 随着虚拟现实 $(\mathrm{VR})$ 技术 ${ }^{[2]}$ 的发展, 采用 VR 技术为仿真系统构造虚拟环境, 提高了 培训场景的真实感。但基于计算机软件的仿真培训技术，不能模拟真实的电力系统环境，体验感和沉浸感 较差, 本文提出的电力设备硬件实物仿真培训系统, 可以提升仿真培训系统的交互体验能力, 增强培训仿 真的体验感和沉浸感，提升培训仿真的智能化水平和实际培训效果。

\section{1 电力安全培训实体仿真系统构成}

电力安全培训实体仿真系统组成如图 1 所示, 包括 $\mathrm{N}$ 个仿真电力设备、操作人员位置和行为动作捕捉 单元、培训系统主机及无线收发单元。培训系统主机和各个仿真电力设备之间通过 ZigBee 无线收发单元 进行数据传输, 实时采集各个仿真电力设备工作状态, 并且利用无线传输网络控制设备内各种故障现象的 发生。培训系统主机利用以太网实时采集位置和行为动作捕捉单元发送的设备上特征点的动态或静态三维 坐标、操作人员各个关节点的三维实时坐标, 并通过和接收到的设备状态监测信息融合, 获得操作人员动 作行为的一致性解释和描述。根据安全操作相关的通用规程和特定业务相关操作规定, 将设备操作转换为 相应的专家知识规则, 依据操作人员动作行为和设备实时状态信息, 对受训人操作的安全性、标准性进行 评价, 并同时模拟故障现象的发生。

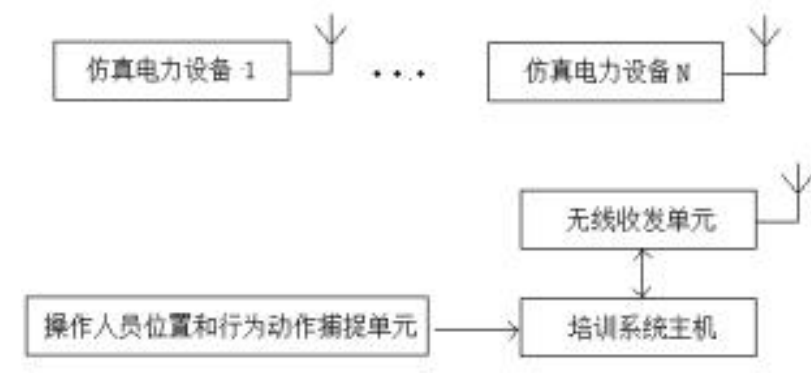

图 1 电力安全培训实体仿真系统组成框图

\section{2 操作人员位置和行为动作捕捉单元}

\section{1 捕捉单元基本组成和原理}

基于立体视觉的设备三维坐标测量及操作人员行为捕捉单元示意图如图 2 所示。在培训现场四周和顶 部适当位置固定放置若干 (图示为 8 只) 带红外照明的智能摄像机，在电力培训设备的各个特征点上固定 红外反光球, 在操作人员工作服上各个关节位置和工作帽上固定红外反光球。摄像机的位置选择原则是保 证每个红外反光球都可以被两个以上摄像机同时捕捉。用同步信号发生器保证各个摄像机采集二维图像的 同步。各个反光球的空间三维坐标从捕捉计算机通过以太网接口实时送到培训系统主机。基于立体视觉的 设备三维坐标测量及操作人员行为捕捉单元工作过程主要包括以下步骤:

(1) 通过对培训现场所有摄像机内外参数标定, 建立三维世界坐标系和摄像机二维坐标系的关系。

（2）在智能摄像机内对图像进行滤波和边缘提取等图像预处理，并精确提取反光球中心坐标。

（3）各个摄像机反光球中心二维坐标通过摄像机以太网接口送到捕捉计算机。

(4) 在捕捉计算机内对各个反光球进行识别, 并对多幅图像中的反光球进行匹配。 
(5) 利用立体视觉三维重建原理 ${ }^{[3]}$ 对反光球在世界坐标系中三维坐标进行重建。

（6）利用改进型卡尔曼滤波预测方法对连续多帧图像中的各个反光球进行跟踪，并记录其运动轨迹。

（7）识别操作设备上位置固定的、保持静止的反射球,记录设备静态坐标位置。通过监测操作设备运 动部件 (如柜门、刀闸等) 上已经固定好的反射球的运动轨迹 (例如, 旋转柜门上的反射球的运动轨迹为 圆弧)，识别运动部件的位置或状态。

(8) 利用各个反射球之间空间相互位置关系以及各个反射球之间距离的约束条件识别工作服上各个 反射球。

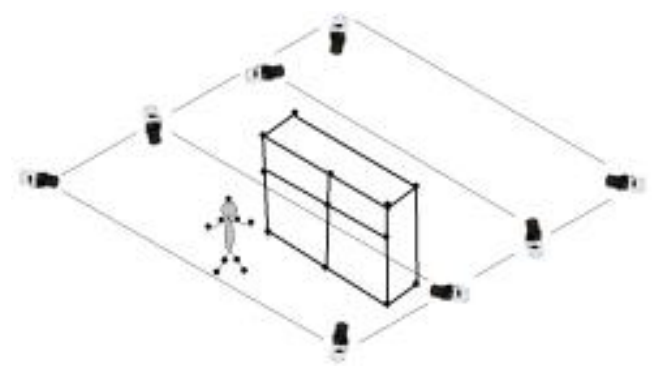

图 2 标记点运动捕捉单元示意图

\section{2 带有标记点的人体骨架建立}

人体的骨架模型主要是由肢体和关节构成，所以进行人体结构描述的关键是进行人体的肢体选择和人 体的关节选取。基于本行为捕捉单元的具体实际情况在操作人员工作服上人员共选取 12 个肢体段（肩、 肘、手、跨、膝、踝、工作帽)。

\section{3 多摄像机标定}

对本系统的多个摄像机通过标定靶标进行标定，将所有摄像机依次编号。先将六个摄像机通过针孔模 型分别进行标定，得到旋转平移矩阵，分别建立与标定靶标坐标系之间的关系。将第一个摄像机的摄像机 坐标系定为世界坐标系, 再通过旋转平移将其他摄像机坐标系, 统一到第一个摄像机坐标系下, 从而得到 各个摄像机与第一个摄像机之间的相对位置关系。而后移动标定靶标的位置, 再通过上述标定过程重新确 定其他 5 个摄像机和 1 号摄像机之间的相对位置，在靶标移动而标定过程中会产生误差，而后建立一个误 差向量最小的目标方程, 取得最优解, 得到其他摄像机相对第一个摄像机之间的相对位置。以此不断修正 误差达到最优标定结果。

标记点轨迹应尽可能地布满每台摄像机的成像平面, 不要集中在某一区域或相对于成像平面轨迹所占 的面积太小。因为标记点轨迹若集中在某一区域, 表示标记点在那一区域有大量重叠, 且那些标记点信息 不能很好的代表整个摄像机成像平面的二维信息, 可能导致摄像机定标参数的不精确。若标记点轨迹能均 匀布满整个成像平面, 将能提高摄像机定标的精度。

\section{4 标记点跟踪}

标记点跟踪是为了使多个标记点在运动以后能一一对应。本运动捕捉系统采用的相机频率为 60 帧/s, 由于人体运动的速度和加速度有限, 反光球在任意两帧之间的运动轨迹可以看成是直线, 其距离可以用任 意两点之间的直线距离公式求得。相邻两个帧间的反光球的速度大小就可以用两帧间直线距离除以相邻两 帧时间得到。相邻的图像帧对应标记点位置的变化非常小，即标记点运动具有很高的连续性。由此我们假 定在相邻的图像中, 标记点运动的速度变化量很小, 且运动轨迹的方向改变很小, 标记点运动轨迹存在平 
滑性。

考虑到实际人体步态行走过程中标记点运动变化差异小, 标记点遮挡、重叠少等特征, 采用的是改进 型的卡尔曼滤波预测跟踪算法[5]。采用卡尔曼滤波在三维空间的运动预测方法, 预测这一时刻的标记点在 下一时刻的位置, 然后根据预测点搜索下一时刻可能的标记点, 并将其作为候选标记点（以预测点到标记 点的距离小于某一固定阈值来判断该标记点是否是候选标记点), 再结合标记点运动轨迹的平滑性约束条 件从候选标记点中初步确定出标记点的位置, 将此位置作为标记点跟踪的初步结果; 同时在发生遮挡, 造 成目标点丢失的情况下, 根据人体各个部位的标记点诸如相互的空间关系, 相互间距离等约束信息, 进一 步确定跟踪的结果。

\section{3 仿真电力设备的组成及其工作原理}

\section{1 仿真电力设备组成}

仿真电力设备组成如图 3 所示, 包括:（1）真实电力设备原机, 包括外壳、面板显示部件（电源指示 灯、状态指示灯、数码管、液晶屏等)、手动操作部件 (按钮、分合开关、拨动控制开关等), 以及设备内 部必要的部件; （2）设备状态监测单元;（3）故障现象模拟单元;（4）显示控制单元;（5）无线收发单 元。

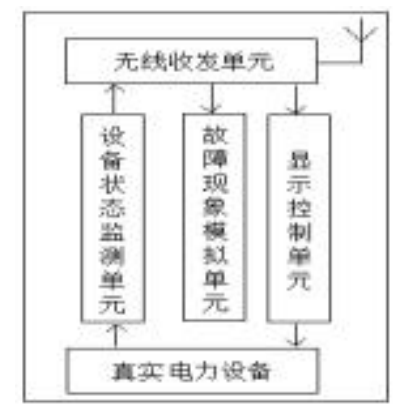

图 3 仿真电力设备组成框图

设备状态监测单元采用传感技术感知设备手动操作部件的工作状态 (如柜门、按钮、分合开关、拨动 控制开关等), 并通过无线收发单元发送到培训系统主机。培训系统主机根据设备工作原理和手动操作部 分的工作状态, 通过无线收发单元发送显示信息到显示控制单元, 控制面板显示内容。培训系统主机根据 设备操作内容对应的专家知识规则[4], 依据操作人员动作行为和设备实时状态信息, 控制柜门自动打开、 着火、烟雾、爆炸和触电等模拟故障现象的发生。

\section{2 电力操作设备实体仿真原理}

图 4 所示为电力操作设备实体仿真结构示意图。 


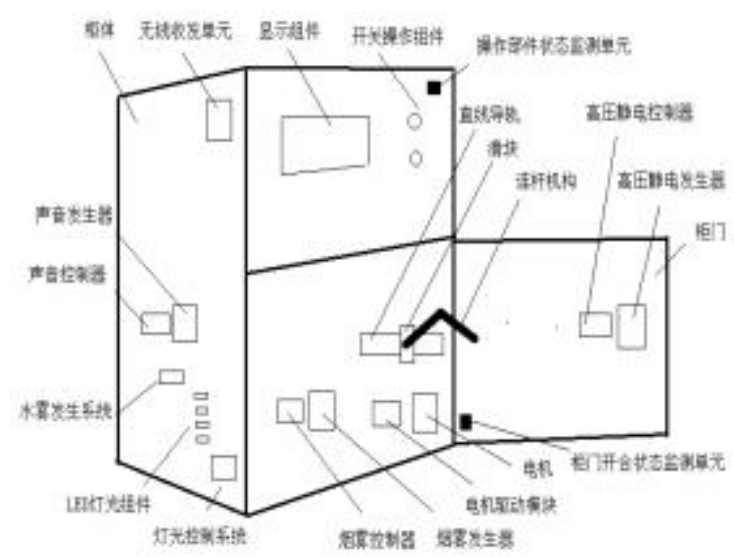

图 4 电力操作设备实体仿真结构示意图

\subsection{1 柜门自动弹开单元}

柜门自动弹开单元由电机驱动模块、电机、直线导轨、滑块、连杆机构等组成。其中直线导轨固定在 设备内壁上, 当电机驱动模块接收到培训系统主机发送的柜门弹开指令时, 驱动电机旋转, 通过直线导轨 上丝杆带动滑块在直线导轨上移动; 连杆机构的一端与滑块相连, 另一端固定在柜门上; 因此, 电机旋转 运动转化为柜门的开启运动过程, 而且通过电机旋转的角度控制柜门打开的程度。

\section{2 .2 着火现象模拟单元}

着火现象模拟单元由水雾发生系统、LED 灯光组件和灯光控制系统组成。水雾发生系统通过超声波雾 化原理产生水雾, 当 LED 灯光组件在灯光控制系统作用下发出不同亮度和颜色的光时, 产生三维立体仿 真火焰, 模拟电力设备内电器着火现象。根据设备内空间结构和容易着火部件的位置, 将水雾发生系统、 LED 灯光组件和灯光控制系统固定在适当位置。

\section{2 .3 着火现象模拟单元}

烟雾现象模拟单元由烟雾发生器和烟雾控制器组成。根据设备内空间结构和容易产生烟雾的位置, 将 烟雾发生器和烟雾控制器固定在适当位置。烟雾发生器可以将无毒的油转化为灰色烟雾, 模拟电力设备内 电器温度过高或着火产生的烟雾。烟雾现象模拟单元如图 5 所示, 当收到培训系统主机的开启信号后（输 入信号高电平), 三极管饱和导通, 继电器线圈通电, 继电器开关闭合, 启动烟雾发生器喷发烟雾。

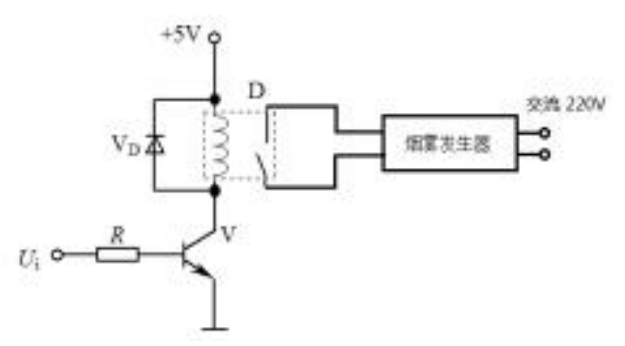

图 5 烟雾发生单元硬件组成

\section{2 .4 着火现象模拟单元}

声音现象模拟单元由声音录放模块、功率放大器和音箱组成, 用来模拟设备内部产生的爆炸、打火等 声音, 可以固定在设备内部空闲位置即可。当设备内无线收发单元收到培训系统主机要求播放某一段录音 时, 通过串行总线控制声音录放模块内部已经录制好的模拟声音的播放。

\section{2 .5 触电现象模拟单元}

触电现象模拟单元由高压静电控制器和高压静电发生器组成, 用来模拟设备内部产生的静电现象, 模 
拟触电的感觉, 静电发生器端子可以固定在门把手等位置。如 6 所示为触电现象模拟单元, 当设备内无线 收发单元收到培训系统主机要求放电的信号时, 发出控制信号使继电器开关 $\mathrm{K}$ 拨向右, 电容上预先充好的 电荷通过电阻、电感放电，使操作人产生触电的感觉。

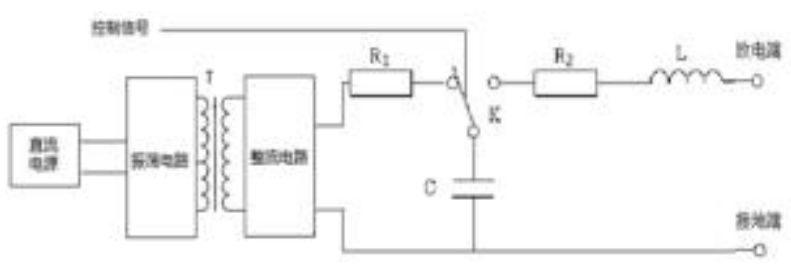

图 6 静电发生器电路

3.2.6 设备状态监测单元

设备状态监测单元采用传感技术 (光电开关、角度编码器等) 感知设备操作部件的工作状态以及柜门 的状态, 并通过无线收发单元发送到培训系统主机。例如: 高压开关柜设备状态信息监测项目包括: 手车 试验位置、手车工作位置、柜门开启与关闭、接地开关合闸与分闸、断路器合闸与分闸、手车在运转车上 是否锁定、转运车与柜体是否锁定、二次插头是否插入和柜外手车操作孔操作状态等。

\section{2 .7 显示控制单元}

显示控制单元代替真实设备内的显示控制部分的功能, 根据设备运行原理、操作部件的状态以及通过 无线收发单元接收的来自培训系统主机的信息，对要显示的内容进行控制，逼真模拟真实设备工作状态。

\subsection{8 无线收发单元}

无线收发单元主要包括无线收发模块和接口模块。无线收发模块实现与主机之间的无线数据通信, 接 口模块实现与显示控制单元、设备状态监测单元和各个故障现象模拟单元之间的互联与有线通信。

\section{4 培训系统软件功能模块}

\section{1 培训管理模块}

培训管理模块实现教案信息、培训人员信息和培训考核结果管理等，建立培训系统数据库。

教案信息包括模型库、动作库和资料库。三维模型库:构建仿真系统中的场景模型、操作设备器件模型、 人物模型等三维模型。动作库: 当操作人员在培训现场进行行走, 操纵仿真实物设备时, 需要将操作人员 的动作与场景融为一体, 建立操作人员行走及各种操作设备动作库。资料库: 资料库主要作为仿真培训系 统的辅助工具。资料库同样具有资料人工添加和批量导入、编辑的功能。

培训人员信息和培训考核结果管理包括操作人员姓名、性别、班组、职责、参加考核日期、操作内容 和考核结果等。

\section{2 数据采集与控制模块}

数据采集与控制模块通过有线方式采集场景和操作设备特征点的三维空间坐标以及操作人员特征关 节点三维坐标信息; 通过无线方式实时采集操作设备状态信息; 通过无线方式控制仿真电力设备产生声音、 烟雾、着火、漏电以及柜门自动弹开等现象。

\section{3 标准化教案生成模块}

培训系统通过记录操作熟练的教练员在完成某项具体操作内容时的行走轨迹、与设备之间的安全距 离、操作动作及设备状态的改变等, 形成标准化操作教案, 作为培训时评价依据。 


\section{4 培训模块}

受训人进行某项操作任务的实际培训, 通过判断操作过程是否符合此项操作任务的标准化操作教案, 对受训人操作的安全性、标准性给出评价。培训模块分为学习模式、实训模式和考试模式。其中学习模式 在操作过程中用培训主机连接的扬声器及时提醒受训人员在操作过程中所犯的错误, 实训模式在评估结束 后进行汇总报错, 而考试模式除了汇总报错外, 还有根据错误多少与错误权重系数对受训人的规范程度自 动打分。

\section{5 结论}

电力安全培训实体仿真系统能有效监测仿真设备状态, 感知操作人员的行为动作, 模拟误操作故障现 象, 生成智能化教案, 对电力操作人员进行实物仿真培训。大大提升仿真培训系统的交互体验能力, 增强 培训的体验感和沉浸感, 提升电力安全培训的智能化水平和实际培训效果。本实验室将进一步进行无标记 点的光学运动捕捉技术的研究, 提高培训的逼真度。研究成果也可以用于类似行业培训领域。

\section{参考文献:}

[1] 吴声声. 电力企业人因安全的研究与应用[D]. 北京: 北京交通大学, 2013.

[2] 周建松. 基于虚拟现实技术的电力培训系统设计[D]. 福州: 福建师范大学, 2013.

[3] 王付新,黄艈瑜,孟偲,等.三维重建中特征点提取算法的研究与实现[J]. 工程图学学报,2007(3):91-93.

[4] 蔡自兴, 徐光祐. 人工智能及其应用 $[\mathrm{M}]$. 北京: 清华大学出版社, 2010.

[5] 高增敏, 王首勇, 郑作虎等. 一种适用于机动目标跟踪的改进卡尔曼滤波器算法[J]. 空军雷达学院学报, 2011,25 (5): $339-342$.

\section{References}

[1] Wu Sheng-sheng: Research and Application on Safety Human Factors in Electricity Enterprises (MS., Beijing:Beijing Jiaotong University, China 2013) .

[2] Zhou Jian-son. Electric training simulation design based virtual reality (MS., Fujian Normal University, China 2013).

[3] Wang Fu-xin, Huang Yu-yu, Meng Cai, et al. "Research and realization of thee feature point extraction algorithm in 3D reconstruction" [J]. Journal of Engineering Graphics, (2007), No.03, p.91-93 (in Chinese).

[4] Cai Zi-xin, Xu Guang-you. Artificial intelligence and its application [M]. Beijing: Tsinghua University press, 2010.

[5] Gao Zeng-min, Wang Shou-yong, Zheng Zuo-hu, et.al. "Improved kalman filter algorithm for maneuvering target tracking'[J]. Journal of Air Force Radar Academy, Vol. 25(2011), No.05, p.339-342 (in Chinese). 